\title{
Morphological Characterisation of Selected Ugandan Sweet potato (Ipomoea batatas L) Varieties for Food and Feed
}

\section{Muinde J Mbithe ${ }^{1 *}$, Runo Steven ${ }^{1}$, Sammy Agili², Musembi B Kivuva ${ }^{3}$, Wambua F Kioko ${ }^{1}$ and Eric Kuria ${ }^{1}$}

${ }^{1}$ Department of Biochemistry and Biotechnology, School of Pure and Applied Sciences, Kenyatta University, P.O. Box 43844-00100, Nairobi, Kenya 2 International Potato Center (CIP-KENYA) Sub-Saharan Africa region, P.O. Box 25171-00603, Nairobi, Kenya

${ }^{3}$ Dry land Research Center, Kenya Agricultural and Livestock Research Organisation (KALRO) P.O Box 340-90100, Machakos, Kenya

\begin{abstract}
Sweet potato Ipomoea batatas L. (Lam.) is a symbol in the fight for a global nutrition plan that can save millions of children and help build a healthier and more productive future. However, characterisation of sweet potato varieties with optimal morphological features suitable for both food and feed has not been done. A population of 10,000 first filial generations (F1) sweet potato lines derived from seeds was generated through polycross crossing design in Uganda using 11 parents. Preliminary evaluation for the suitability of dual use of the F1's led to selection of 11 varieties which were the basis of this study. This study therefore sought to morphologically characterise selected Ugandan sweet potato varieties to identify those with superior characteristics suitable for food and feed purposes. Sweet potato plants were raised from seeds after scarification. A selection of seedlings possessing single leaf lobes was done, after which they entered observation yield trials (OT). This was done in order to discard those that clearly did not meet the lowest acceptable gross morphological descriptors. The data were subjected to analysis of variance in order to find out if significant differences exist between the varieties based on morphological characterization. Cluster analysis was done using Minitab version 17 software. This study enables the selection of sweet potato varieties with optimal characteristics for both food and feed use. The data generated from this study could help recommend to farmers on how dual-purpose sweet potato could be produced, in order to provide enough healthier food to millions of children in Uganda and in the world, and better feed for live-stock farmers.
\end{abstract}

Keywords: Sweet potato; Morphological characterization

\section{Introduction}

Food security is the access by all people at all times to enough food for an active, healthy life. In this quest, mixed crop-livestock systems are important in overcoming the agricultural production challenges of smallholder farmers in the developing countries of sub-Saharan Africa [1]. In most cases, cereals provide the cheapest source of food for many people, but with increasing populations and decreasing farm sizes in the region, alternative staple foods have been sought, especially sweet potato (Ipomoea batatas) [2].

Through characterization, diversity that exists in a germplasm population can be estimated and effectively studied [3]. Morphological characterization in sweet potato is done by assessing variations in the vine, leaf, flower and storage root characteristics [4]. This method has been used previously for identifying sweet potato cultivars, duplicate accessions, detecting unique character traits and correlation with characteristics of agronomic importance [5]. Standard descriptor lists provide an international format, thereby producing a universally understood language for plant genetic resource data [6]. Morphological and agronomic characters, such as the storage root to vine ratio, have been used to identify and select dual-purpose sweet potato varieties [7].

Sweet potato storage roots are rich in high calorie starch, with more amylose than amylopectin, a rich source of dietary fibre, minerals, antioxidants, flavonoid phenolic compounds such as beta-carotene and vitamin A [8]. Other vitamins include vitamin B6, B1, niacin and riboflavin. This crop lacks saturated fats or cholesterol, making it a recommendable health food even to diabetics [9]. Moreover, its leaves are rich in proteins, iron, potassium, sodium, vitamin $\mathrm{C}$, vitamin $\mathrm{K}$, folates, minerals, fat, fibre, and carbohydrates. Leaves have low levels of toxicants, except for oxalate, which can be reduced by cooking [10].

Most small holder farmers practice mixed farming due to scarcity of land. In these systems there is evidence that the amount of fodder available for livestock is inadequate in both quality and quantity [11]. Dual-purpose sweet potato is thus increasingly becoming an important crop in maize-based farming systems where it can be planted during the off-season to cater for food security [12]. Its vines are also used as livestock feed due to the increasing scarcity of feeds. Sweet potato is economically viable, as seen in the relatively high yields, net returns, and crude protein content of the fodder, which increases milk production and incomes [13].

Malnutrition due to deficiency of micronutrients in the diet affects the health of over half the world's population. Vitamin A deficiency in particular has become a major concern since it can cause blindness and weaken the body's immune system in humans. Vitamin A deficiency is more severe in sub-Saharan Africa, where an estimated 32\% of preschool aged children are affected. In addition to these health and nutritional challenges, land available for natural grazing is rapidly diminishing due to the increasing human population. The value of maize stock as livestock feed is, as with other crop residues, is limited by its poor digestibility and low crude-protein and mineral content. There is, therefore, a need for a crop that can be efficiently used for both food and feed, such as the sweet potato. Sweet potato grows in marginal conditions, requires little labour and little or no chemical fertilisers. It is a cheap, nutritious solution for developing countries, which need to grow more food on less area to sustain rapidly growing populations. It also provides inexpensive, high-protein fodder for animals. This study

*Corresponding author: Muinde Jane Mbithe, Department of Biochemistry and Biotechnology, School of Pure and Applied Sciences, Kenyatta University, P.O. Box 43844-00100, Nairobi, Kenya, Tel: +254718507667 E-mail:m.muinde9@students.ku.ac.ke

Received February 07, 2016; Accepted March 24, 2016; Published March 31 2016

Citation: Mbithe MJ, Steven R, Agili S, Kivuva MB, Kioko WF, et al. (2016) Morphological Characterisation of Selected Ugandan Sweet potato (Ipomoea batatas L) Varieties for Food and Feed. J Phylogen Evolution Biol 4: 163 doi:10.4172/2329-9002.1000163

Copyright: (c) 2016 Mbithe MJ, et al. This is an open-access article distributed under the terms of the Creative Commons Attribution License, which permits unrestricted use, distribution, and reproduction in any medium, provided the original author and source are credited. 
Citation: Mbithe MJ, Steven R, Agili S, Kivuva MB, Kioko WF, et al. (2016) Morphological Characterisation of Selected Ugandan Sweet potato (Ipomoea batatas L) Varieties for Food and Feed. J Phylogen Evolution Biol 4: 163. doi:10.4172/2329-9002.1000163

Page 2 of 6

thus aimed at characterizing sweet potato varieties that can be used for both food and feed under the agricultural context of Uganda.

\section{Materials and Methods}

\section{Plant materials}

Sweet potato F1 lines generated from a polycross of 11 Ugandan sweet potato parents were analysed in this study. The crossing was done at the International Potato Center (CIP-Uganda). The parents were: Rwabuganda, Kyebandula, Naspot1, Kyabafuruki, Magabari, NaspotII, New Kawogo, Dimbukabukulula, Kigabali, BND145L and Shock. The seeds were germinated at KALRO-Kiboko and the seedlings transplanted into the experimental plot after establishment.

\section{Scarification of sweet potato seeds}

Sweet potato seeds do not have a dormancy period but maintain their viability for many years. Seed germination is therefore difficult and requires scarification by mechanical abrasion or chemical treatment [4]. In this case, a chemical treatment was done for scarification using concentrated sulphuric (VI) acid. Seeds were placed in plastic mesh and submerged in concentrated sulphuric (VI) acid until the acid started turning dark (10 $\mathrm{min}-40 \mathrm{~min}$ ). They were then rinsed in running water. After scarification, the seeds were planted in seedling trays containing sterile soil, watered and placed under net tunnel shade. The trays were kept moist until seedling establishment. Before transplanting, the seedlings were hardened by reducing the moisture of the soil in the seedling trays.

\section{Screening of dual-purpose varieties}

A selection on single true seed plant exhibiting dual-purpose characteristics based on leaf lobes was done. The selected plants were multiplied and planted in the field organised according to their varieties on mounds $1 \mathrm{~m}$ apart, with $30 \mathrm{~cm}$ between plants and the experiment replicated three times. Observation yield trials (OTs) were carried out in order to discard those which clearly did not meet a lowest acceptable value of the given descriptors for dual-purpose (food and feed) varieties, as shown in Table 1.

\section{Collection of morphological characters}

Morphological characterisation of the 11 sweet potato varieties based on 18 vine and leaf characters and 4 storage root descriptors was done at 90 days and 180 days after planting (DAP). It was done using international descriptors for sweet potato developed by CIP (international potato center) [6] (Table 1).

\section{Data analysis}

The proximate data was analyzed using Analysis Of Variance (ANOVA) followed by Tukey's post hoc statistical tools as implemented in the statistical package for social sciences (SPSS) Version 20 software package. Minitab version 17.0 software (State College Pennsylvania,

\begin{tabular}{|c|c|c|}
\hline Morphology & Acceptable description & CIP score \\
\hline Twining & Moderately twining & 5 \\
\hline Plant Type & Spreading $(151 \mathrm{~cm}-250 \mathrm{~cm})$ & 7 \\
\hline Ground Cover & High $(75 \%-90 \%)$ & 7 \\
\hline Vine Internode & $\begin{array}{l}\text { (i) Length-Intermediate }(6 \mathrm{~cm}-9 \mathrm{~cm}) \\
\text { (ii) Diameter-Thick }(10 \mathrm{~mm}-12 \mathrm{~mm})\end{array}$ & 5 \\
\hline Vine Pigmentation & $\begin{array}{l}\text { (1) Predominant vine color - Green } \\
\text { (ii) Secondary vine color-Absent }\end{array}$ & 1 \\
\hline Vine Tip Pubescence & Sparse & 3 \\
\hline Mature Leaf Shape & Either: (1) Rounded (2) Reniform (3) Cordate (4) Tiangular & $1,2,3,4$ \\
\hline Leaf Lobes Type & No lateral lobes or Very slight (teeth) & 0,1 \\
\hline Leaf Lobe Number & 1 & 1 \\
\hline Shape of Central Leaf Lobe & Absent (ii) toothed & 0,1 \\
\hline Mature Leaf Size & 1. Large $(16 \mathrm{~cm}-25 \mathrm{~cm})$ & 7 \\
\hline Abaxial Leaf Vein Pigmentation & Green & 2 \\
\hline Foliage Color & $\begin{array}{ll}\text { (i) } & \text { Mature leaf color-Green } \\
\text { (ii) } & \text { Immature leaf color-Green }\end{array}$ & $\begin{array}{l}2 \\
2\end{array}$ \\
\hline Petiole Length & Intermediate $(21 \mathrm{~cm}-30 \mathrm{~cm})$ & 5 \\
\hline Petiole Pigmentation & Green & 1 \\
\hline Storage Root & $\begin{array}{l}\text { (1) Storage root shape; Round elliptic, Elliptic, Long-Elliptic } \\
\text { (ii) Storage root cortex thickness-Intermediate }(3 \mathrm{~mm}) \\
\text { (iii) Storage root skin color } \\
\text { a. Predominant skin color-Any } \\
\text { b. secondary skin color-Absent } \\
\text { (iv) Storage root flesh color } \\
\text { a. Predominant flesh color-Any } \\
\text { b. Secondary flesh color; Either: Absent. White, cream, yellow, orange. }\end{array}$ & $\begin{array}{l}2,3,8 \\
5 \\
1-9 \\
0 \\
1-9 \\
0-4\end{array}$ \\
\hline
\end{tabular}

Table 1: Vegetative and storage roots characteristics used for evaluation of sweet potato varieties for food and feed use (CIP, AVRDC, IBPGR (1991)). 
Citation: Mbithe MJ, Steven R, Agili S, Kivuva MB, Kioko WF, et al. (2016) Morphological Characterisation of Selected Ugandan Sweet potato (Ipomoea batatas L) Varieties for Food and Feed. J Phylogen Evolution Biol 4: 163. doi:10.4172/2329-9002.1000163

Page 3 of 6

USA) was used to carry out cluster analyses and principal component analysis. Cluster analyses based on morphological parameters yielded dendrograms that were used to examine the phenotypic relatedness among the 11 sweet potato varieties. Principal Component Analysis (PCA) was carried out to determine the major determinants of variation and correlation based on the 18 descriptors among the varieties.

\section{Results}

\section{Vine characters}

Analysis of variance indicated highly significant differences ( $\mathrm{p}$ $\leq 0.001)$ in most of the vine characters. However, vine internode length and vine tip pubescence were not significantly different $(\mathrm{p} \geq$ 0.05 ). Among the 11 varieties, twining (TW) was highest in Naspot II (5.6) and lowest in Shock (3.8). Plant type (PT) ranged between 5.2 (Shock) and 7.0 (New-Kawogo). Groundcover (GC) was highest in Kigabali (7.1) and lowest in Naspot I (6.3).Vine internode length (VIL) ranged between 3.0 and 3.7 among the varieties. Node diameter (ND) was highest in NaspotII (4.7) and lowest in NaspotI (3.4).Vine pigmentation (VPG) ranged between 1.1 and 3.4 among the varieties .Vine tip pubescence (VTP) ranged between 1.9 and 3.1 among the11 varieties. Vine weight was highest in Kigabali (2.9) and lowest in Kyebandula and Naspot I (1.0) (Table 2).

\section{Leaf characters}

Among the 11 varieties, analysis of variance indicated highly significant differences $(\mathrm{p} \leq 0.001)$ in the leaf characters, as shown in Table 3. Leaf Outline (LOT) ranged between 3.2 and 3.9 while abaxial pigmentation (ABPG) ranged between 3.4 and 5.4. Immature leaf pigmentation (IMLPG) was highest in Shock (5.1) and lowest in Naspot I (2.0). New Kawogo had the highest petiole length (PTL), while Kyebandula had the lowest. Petiole pigmentation varied most significantly, with Kigabali being the highest (4.1) while Rwabuganda was the lowest (1.5) (Table 3).

\section{Root characters}

Among the 11 varieties, all the root characters evaluated showed significant differences ( $\mathrm{p} \leq 0.05$, Table 4 ). Root shape varied between 2.5 and 6.1, while cortex thickness was highest in Newkawogo (6.8) and lowest in Kyebandula (4.7).Among the 11 varieties, flesh colour ranged between 1.4 and 3.7. Root fresh weight was highest in New Kawogo (2.8 $\mathrm{kg} /$ plant) and lowest in Kyebandula (0.9 kg/plant).

\section{Comparison of morphological parameters in the various varieties}

Figure 1 show a dendrogram constructed from data sets containing the mean values of all 18 morphological descriptors. It shows that the varieties separated into two super clusters, A and B. Super cluster A is composed of two sub-clusters, I and II, each subdividing further into two other sub-sub-clusters, Ia, Ib, IIa and IIb, respectively, while super cluster B is composed of Kigabali and New Kawogovarieties. Sub-subcluster Ia included Naspot I and Rwabuganda varieties, while sub-subcluster Ib contained Dimbukabukulula, Magabari and Kyabafuruki varieties. Sub-sub-cluster IIa grouped together BND145L and Kyebandula, while sub-sub-cluster IIb included Shock and NaspotII varieties.

\section{Principal component analysis (PCA) based on morphological traits}

PCA was performed for all the 18 morphological traits among the 11 sweet potato varieties, as indicated in Table 5 . Out of the 18 traits, 4 principal components exhibited more than one Eigen value and showed about $78.2 \%$ variability among the characters under investigation.PC1

\begin{tabular}{|c|c|c|c|c|c|c|c|c|}
\hline \multirow[b]{2}{*}{ Variety } & \multicolumn{8}{|c|}{ Vine characteristics } \\
\hline & TW (score: 0-9) & PT (score: 3-9) & GC (score: 3-9) & $\begin{array}{c}\text { VIL (score: } \\
1-9)\end{array}$ & ND (score: 1-9) & VPG (score: 1-9) & $\begin{array}{c}\text { VTP (score: } \\
0-7 \text { ) }\end{array}$ & VWT (kg/plant) \\
\hline Naspot 1 & $5.2 \pm 0.47^{\mathrm{bc}}$ & $5.9 \pm 0.36^{\mathrm{abc}}$ & $6.3 \pm 0.30^{\mathrm{a}}$ & $3.4 \pm 0.18^{a}$ & $3.4 \pm 0.31^{\mathrm{a}}$ & $1.6 \pm 0.41^{a}$ & $3.0 \pm 0.28^{\mathrm{a}}$ & $1.0 \pm 0.09^{a}$ \\
\hline BND 145L & $4.3 \pm 0.36^{\text {ab }}$ & $5.5 \pm 0.24^{\mathrm{ab}}$ & $6.8 \pm 0.13^{\mathrm{ab}}$ & $3.5 \pm 0.24^{a}$ & $3.4 \pm 0.18^{\mathrm{a}}$ & $1.7 \pm 0.25^{\mathrm{a}}$ & $2.5 \pm 0.41^{a}$ & $1.5 \pm 0.30^{\mathrm{ab}}$ \\
\hline Shock & $3.8 \pm 0.22^{\mathrm{a}}$ & $5.2 \pm 0.13^{\mathrm{a}}$ & $6.9 \pm 0.10^{\mathrm{ab}}$ & $3.0 \pm 0.00^{\mathrm{a}}$ & $4.0 \pm 0.22^{2 \mathrm{abc}}$ & $1.4 \pm 0.27^{\mathrm{a}}$ & $2.9 \pm 0.46^{a}$ & $1.8 \pm 0.19^{\mathrm{abc}}$ \\
\hline Dibuka Bukulula & $5.1 \pm 0.23^{\mathrm{bc}}$ & $6.2 \pm 0.22^{2 \mathrm{abc}}$ & $6.8 \pm 0.13^{\mathrm{ab}}$ & $3.4 \pm 0.18^{\mathrm{a}}$ & $3.6 \pm 0.21^{\mathrm{ab}}$ & $1.2 \pm 0.17^{\mathrm{a}}$ & $2.6 \pm 0.41^{\mathrm{a}}$ & $2.4 \pm 0.36^{\mathrm{bc}}$ \\
\hline Rwabuganda & $5.0 \pm 0.00^{\mathrm{bc}}$ & $6.1 \pm 0.30^{\mathrm{abc}}$ & $6.9 \pm 0.10^{\mathrm{ab}}$ & $3.0 \pm 0.00^{\mathrm{a}}$ & $3.5 \pm 0.19^{\mathrm{ab}}$ & $1.1 \pm 0.15^{\mathrm{a}}$ & $1.9 \pm 0.43^{\mathrm{a}}$ & $2.5 \pm 0.36^{\mathrm{bc}}$ \\
\hline Kigabali & $5.2 \pm 0.14^{\mathrm{bc}}$ & $6.1 \pm 0.27^{\mathrm{abc}}$ & $7.1 \pm 0.10^{\mathrm{b}}$ & $3.5 \pm 0.24^{\mathrm{a}}$ & $4.1 \pm 0.22^{\mathrm{abc}}$ & $3.4 \pm 0.38^{c}$ & $2.7 \pm 0.29^{\mathrm{a}}$ & $2.9 \pm 0.49^{c}$ \\
\hline New Kawogo & $5.5 \pm 0.19^{c}$ & $7.0 \pm 0.00^{\circ}$ & $7.0 \pm 0.00^{\mathrm{b}}$ & $3.6 \pm 0.21^{\mathrm{a}}$ & $4.0 \pm 0.27^{\mathrm{abc}}$ & $3.2 \pm 0.37^{\mathrm{bc}}$ & $2.7 \pm 0.44^{\mathrm{a}}$ & $2.4 \pm 0.25^{\mathrm{bc}}$ \\
\hline Magabari & $5.5 \pm 0.19^{c}$ & $6.4 \pm 0.25^{\text {bc }}$ & $7.0 \pm 0.00^{b}$ & $3.5 \pm 0.32^{\mathrm{a}}$ & $4.5 \pm 0.19^{b c}$ & $1.9 \pm 0.30^{\mathrm{ab}}$ & $2.7 \pm 0.40^{\mathrm{a}}$ & $2.7 \pm 0.21^{\mathrm{bc}}$ \\
\hline Naspot II & $5.6 \pm 0.21^{c}$ & $6.0 \pm 0.22^{\mathrm{abc}}$ & $6.7 \pm 0.16^{\mathrm{ab}}$ & $3.7 \pm 0.21^{\mathrm{a}}$ & $4.7 \pm 0.21^{\circ}$ & $2.3 \pm 0.30^{\mathrm{abc}}$ & $3.1 \pm 0.40^{\mathrm{a}}$ & $1.9 \pm 0.19^{\mathrm{abc}}$ \\
\hline Kyabafuruki & $5.0 \pm 0.00^{\mathrm{bc}}$ & $6.4 \pm 0.21^{\mathrm{bc}}$ & $6.7 \pm 0.15^{\mathrm{ab}}$ & $3.0 \pm 0.00^{\mathrm{a}}$ & $3.8 \pm 0.24^{\mathrm{abc}}$ & $1.6 \pm 0.35^{\mathrm{a}}$ & $2.6 \pm 0.38^{\mathrm{a}}$ & $1.9 \pm 0.23^{\mathrm{abc}}$ \\
\hline Kyembadula & $5.0 \pm 0.00^{b c}$ & $5.3 \pm 0.20^{\mathrm{ab}}$ & $6.7 \pm 0.17^{\mathrm{ab}}$ & $3.0 \pm 0.00^{\mathrm{a}}$ & $3.5 \pm 0.22^{\mathrm{ab}}$ & $2.0 \pm 0.36^{\mathrm{ab}}$ & $2.4 \pm 0.46^{a}$ & $1.0 \pm 0.14^{a}$ \\
\hline
\end{tabular}

Key: TW - Twinning, PT - Plant Type, GC - Ground Cover, VIL - Vine Inter-Node Length, ND - Node Diameter, VPG - Vine Pigmentation, VTP - Vine Tip Pubescence, VWT - Vine Weight Means of sweet potato varieties under field conditions followed by the same letters within a column are not significantly different, Tukey's post hoc ( $p$ $\leq 0.05$ ) 
Citation: Mbithe MJ, Steven R, Agili S, Kivuva MB, Kioko WF, et al. (2016) Morphological Characterisation of Selected Ugandan Sweet potato (Ipomoea batatas L) Varieties for Food and Feed. J Phylogen Evolution Biol 4: 163. doi:10.4172/2329-9002.1000163

Page 4 of 6

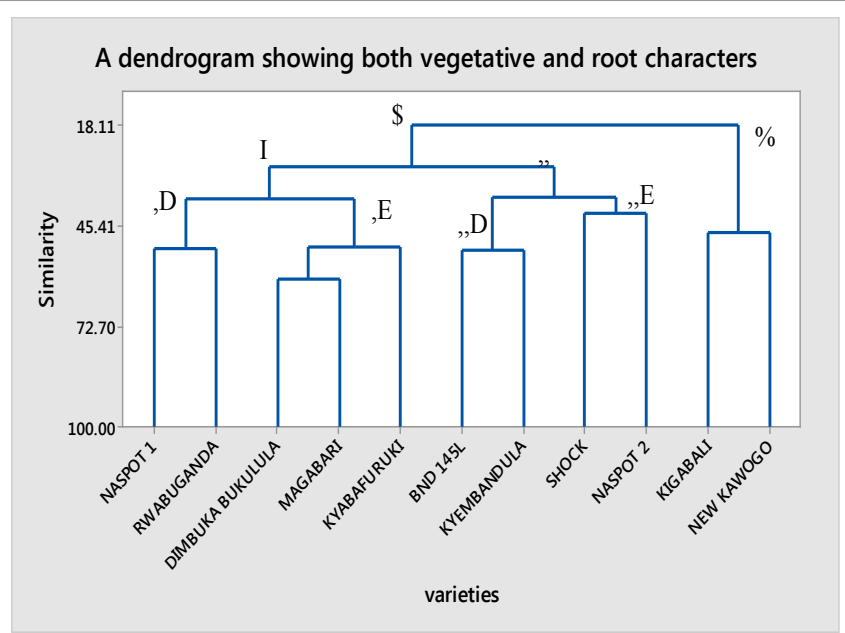

Figure 1: UPGMA dendrogram, based on Euclidean distance coefficient, of 11 varieties generated using morphological characters. showed $36.9 \%$, PC2 showed $18.9 \%$, PC3 had $12.4 \%$ and PC4 indicated $10 \%$ variability among the varieties for the characters being studied. Principal component one (PC1), Principal component two (PC2), Principal component three (PC3), and Principal component four (PC4) had Eigen values of 6.64, 3.40, 2.23 and 1.7938, respectively (Table 5, Appendix1). Further, PC1 was highly and positively correlated to all the traits except leaf outline, root shape and root flesh colours, which were negatively correlated. Most of the traits for PC2 were negatively correlated, with only 8 characters positively correlated, as shown in Table 5. PC3 exhibited very high positive correlation in all the traits, apart from abaxial pigmentation, petiole pigmentation and root shape, which were negatively correlated. On the other hand, PC4 showed high positive correlation between plant type, ground cover, vine pigmentation, vine weight, leaf outline, abaxial pigmentation and petiole length. However, it was highly and negatively correlated to the other 11 characters under investigation, as shown in Table 5.

\begin{tabular}{|c|c|c|c|c|c|}
\hline \multirow[b]{2}{*}{ Variety } & \multicolumn{2}{|c|}{ Leaf characteristics } & \multirow[b]{2}{*}{ IMLPG } & \multirow[b]{2}{*}{ PTL } & \multirow[b]{2}{*}{ PTPG } \\
\hline & LOT & ABPG & & & \\
\hline & (Score: 1-7) & (Score: 1-9) & (Score: 1-9) & (Score: 1-9) & (Score: 1-9) \\
\hline Naspot 1 & $3.6 \pm 0.10^{\mathrm{abcd}}$ & $3.4 \pm 0.45^{\mathrm{a}}$ & $2.0 \pm 0.05^{\mathrm{a}}$ & $4.7 \pm 0.21^{\mathrm{ab}}$ & $2.1 \pm 0.51^{\mathrm{ab}}$ \\
\hline BND 145L & $3.7 \pm 0.10^{\mathrm{bcd}}$ & $5.3 \pm 0.53^{a}$ & $3.4 \pm 0.41^{\mathrm{abc}}$ & $5.0 \pm 0.29^{a b c}$ & $2.3 \pm 0.44^{\mathrm{abc}}$ \\
\hline Shock & $3.8 \pm 0.91^{\mathrm{cd}}$ & $4.2 \pm 0.50^{a}$ & $5.1 \pm 0.44^{c}$ & $5.8 \pm 0.30^{\mathrm{bc}}$ & $2.1 \pm 0.43^{b c}$ \\
\hline Dibuka Bukulula & $3.9 \pm 0.11^{d}$ & $4.3 \pm 0.51^{\mathrm{a}}$ & $4.0 \pm 0.61^{\mathrm{bc}}$ & $4.9 \pm 0.33^{\mathrm{ab}}$ & $2.0 \pm 0.35^{\mathrm{ab}}$ \\
\hline Rwabuganda & $3.5 \pm 0.11^{\mathrm{abcd}}$ & $3.7 \pm 0.50^{\mathrm{a}}$ & $2.4 \pm 0.27^{\mathrm{ab}}$ & $5.0 \pm 0.35^{\mathrm{abc}}$ & $1.5 \pm 0.25^{\mathrm{abc}}$ \\
\hline Kigabali & $3.3 \pm 0.10^{\mathrm{ab}}$ & $5.4 \pm 0.51^{a}$ & $3.1 \pm 0.39^{\mathrm{ab}}$ & $5.7 \pm 0.33^{\mathrm{bc}}$ & $4.1 \pm 0.42^{\mathrm{bc}}$ \\
\hline New Kawogo & $3.2 \pm 0.91^{\mathrm{a}}$ & $5.4 \pm 0.44^{\mathrm{a}}$ & $3.4 \pm 0.45^{\mathrm{abc}}$ & $6.4 \pm 0.32^{c}$ & $5.3 \pm 0.45^{c}$ \\
\hline Magabari & $3.3 \pm 0.10^{\mathrm{ab}}$ & $5.2 \pm 0.44^{a}$ & $2.8 \pm 0.42^{\mathrm{bc}}$ & $5.3 \pm 0.36^{\mathrm{bc}}$ & $2.2 \pm 0.43^{\mathrm{bc}}$ \\
\hline Naspot II & $3.4 \pm 0.11^{\mathrm{abc}}$ & $5.8 \pm 0.47^{a}$ & $4.3 \pm 0.60^{\mathrm{bc}}$ & $5.1 \pm 0.30^{\mathrm{abc}}$ & $2.9 \pm 0.51^{\mathrm{abc}}$ \\
\hline Kyabafuruki & $3.5 \pm 0.12^{\mathrm{abcd}}$ & $3.2 \pm 0.46^{\mathrm{a}}$ & $2.6 \pm 0.36^{\mathrm{ab}}$ & $4.6 \pm 0.37^{\mathrm{ab}}$ & $1.8 \pm 0.52^{\mathrm{ab}}$ \\
\hline Kyembadula & $3.6 \pm 0.11^{\mathrm{bcd}}$ & $4.7 \pm 0.62^{\mathrm{a}}$ & $2.7 \pm 0.40^{\mathrm{ab}}$ & $3.7 \pm 0.25^{\mathrm{a}}$ & $3.3 \pm 0.65^{\mathrm{a}}$ \\
\hline
\end{tabular}

Means followed by the same letters within a column are not significantly different, Tukey's test $(\mathrm{p} \leq 0.05)$

Key: LOT - Leaf Outline, ABPG - Abaxial Leaf Pigmentation, MLPG - Mature Leaf Pigmentation, IMLPG - Immature Leaf Pigmentation, PTL - Petiole Length, PTPG Petiole Pigmentation.

Table 3: Mean scores of leaf characteristics of sweet potato varieties under field conditions.

\begin{tabular}{|c|c|c|c|c|c|}
\hline & \multicolumn{2}{|c|}{ Root characteristics } & \multirow[b]{2}{*}{ Skin colour (Score: 1-9) } & \multirow[b]{2}{*}{ Flesh colour (Score: 1-9) } & \multirow[b]{2}{*}{ Fresh weight (kg/plant) } \\
\hline & Shape (Score: 1-9) & $\begin{array}{l}\text { Cortex thickness (Score: } \\
1-9)\end{array}$ & & & \\
\hline \multicolumn{6}{|l|}{ Variety } \\
\hline Naspot 1 & $5.4 \pm 0.62^{b}$ & $6.1 \pm 022^{\mathrm{ab}}$ & $3.8 \pm 0.59^{\mathrm{ab}}$ & $2.3 \pm 0.36^{a}$ & $1.8 \pm 0.16^{a b c}$ \\
\hline BND 145L & $4.1 \pm 0.60^{\mathrm{ab}}$ & $5.6 \pm 0.29^{a b}$ & $6.3 \pm 0.52^{\mathrm{b}}$ & $1.4 \pm 0.11^{a}$ & $1.0 \pm 0.13^{a}$ \\
\hline Shock & $4.9 \pm 0.67^{\mathrm{ab}}$ & $5.9 \pm 0.44^{\mathrm{ab}}$ & $5.8 \pm 0.56^{b}$ & $2.4 \pm 0.21^{\mathrm{a}}$ & $1.6 \pm 0.14 a b$ \\
\hline Dimbuka Bukulula & $2.9 \pm 0.42^{\mathrm{ab}}$ & $6.1 \pm 0.30^{\mathrm{ab}}$ & $5.2 \pm 0.51^{\mathrm{ab}}$ & $1.9 \pm 0.12^{\mathrm{a}}$ & $1.7 \pm 0.15 a b$ \\
\hline Rwabuganda & $4.9 \pm 0.72^{\mathrm{ab}}$ & $6.1 \pm 0.33^{\mathrm{ab}}$ & $4.2 \pm 0.45^{\mathrm{ab}}$ & $3.7 \pm 0.49^{b}$ & $2.7 \pm 0.25^{\mathrm{cd}}$ \\
\hline Kigabali & $4.1 \pm 0.61^{\mathrm{ab}}$ & $6.3 \pm 0.33^{b}$ & $3.1 \pm 0.46^{a}$ & $1.7 \pm 0.09^{a}$ & $2.2 \pm 0.33^{\mathrm{bcd}}$ \\
\hline New Kawogo & $3.1 \pm 0.5^{\mathrm{ab}}$ & $6.8 \pm 0.24^{b}$ & $5.3 \pm 0.59^{a b}$ & $2.0 \pm 0.05^{\mathrm{a}}$ & $2.8 \pm 0.22^{\mathrm{d}}$ \\
\hline Magabari & $3.0 \pm 0.5^{\mathrm{ab}}$ & $6.0 \pm 0.27^{\mathrm{ab}}$ & $4.2 \pm 0.54^{\mathrm{ab}}$ & $2.1 \pm 0.23^{a}$ & $2.0 \pm 0.16^{\mathrm{bcd}}$ \\
\hline Naspot II & $6.1 \pm 0.22^{\mathrm{ab}}$ & $6.1 \pm 0.27^{\mathrm{ab}}$ & $6.0 \pm 0.57^{b}$ & $2.5 \pm 0.38^{\mathrm{ab}}$ & $1.8 \pm 0.19^{a b c}$ \\
\hline Kyabafuruki & $2.5 \pm 0.3^{\mathrm{a}}$ & $5.7 \pm 0.51^{\mathrm{ab}}$ & $3.2 \pm 0.53^{\mathrm{a}}$ & $2.2 \pm 0.31^{a}$ & $1.5 \pm 0.20^{\mathrm{ab}}$ \\
\hline Kyebandula & $4.3 \pm 0.75^{\mathrm{ab}}$ & $4.7 \pm 0.30^{\mathrm{a}}$ & $5.0 \pm 0.53^{\mathrm{ab}}$ & $2.3 \pm 0.33^{a}$ & $0.9 \pm 0.15^{a}$ \\
\hline
\end{tabular}

Means followed by the same letters within a column are not significantly different, Tukey's test $(p \leq 0.05)$

Table 4: Mean score of tuber characteristics among the varieties under field conditions. 


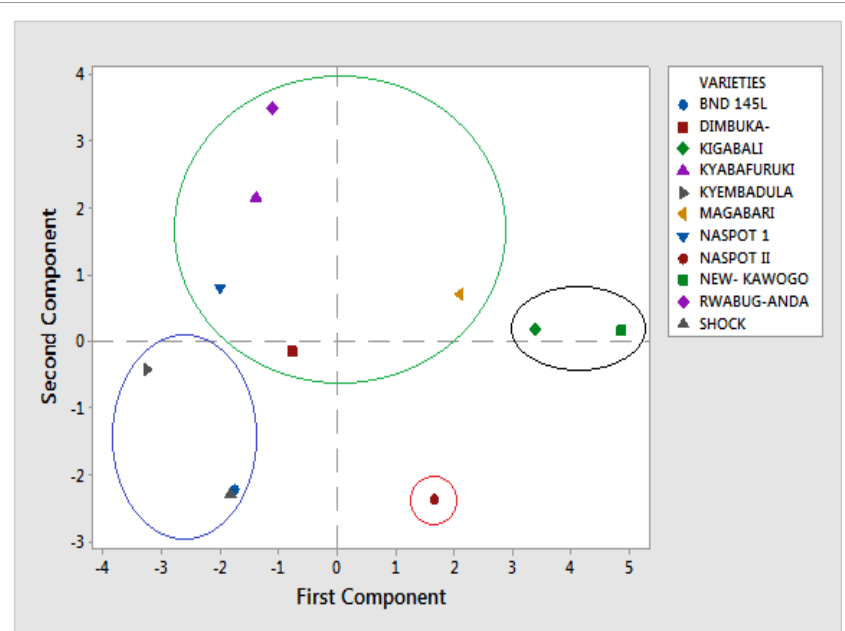

Figure 2: Clustering of 11 sweet potato varieties based on 18 vegetative and root.

\begin{tabular}{|c|c|c|c|c|}
\hline & PC1 & PC2 & PC3 & PC4 \\
\hline Eigen Value & 6.6493 & 3.4072 & 2.2315 & 1.7938 \\
\hline$\%$ Total Variance & 36.9 & 18.9 & 12.4 & 10 \\
\hline$\%$ Cumulative & 36.9 & 55.9 & 68.3 & 78.2 \\
\hline Traits & \multicolumn{4}{|c|}{ Eigenvectors } \\
\hline Twining & 0.226 & 0.191 & 0.371 & -0.136 \\
\hline Plant type & 0.286 & 0.267 & 0.061 & 0.011 \\
\hline Ground cover & 0.236 & 0.002 & 0.385 & 0.318 \\
\hline Vine internode length & 0.268 & -0.214 & 0.217 & -0.102 \\
\hline Node diameter & 0.255 & -0.154 & 0.018 & -0.198 \\
\hline Vine pigmentation & 0.307 & -0.098 & 0.228 & 0.157 \\
\hline Vine tip pubescence & 0.098 & -0.326 & 0.229 & -0.26 \\
\hline Vine weight & 0.28 & 0.165 & 0.325 & 0.096 \\
\hline Leaf outline & -0.304 & -0.153 & 0.211 & 0.03 \\
\hline Abaxial pigmentation & 0.244 & -0.324 & -0.059 & 0.114 \\
\hline Immature leaf pigmentation & 0.048 & -0.405 & 0.371 & -0.078 \\
\hline Petiole length & 0.296 & -0.086 & 0.311 & -0.083 \\
\hline Petiole pigmentation & 0.274 & -0.121 & -0.178 & 0.185 \\
\hline Root shape & -0.082 & -0.179 & -0.07 & -0.565 \\
\hline Root cortex- thickness & 0.295 & 0.078 & 0.167 & -0.262 \\
\hline Root skin colour & 0.042 & -0.413 & 0.176 & -0.078 \\
\hline Root flesh colour & -0.084 & 0.289 & 0.197 & -0.454 \\
\hline Root weight & 0.271 & 0.269 & 0.192 & -0.263 \\
\hline
\end{tabular}

Table 5: Eigen vectors, Eigen values, total variance and cumulative variance among 11 sweet potato varieties based on 18 morphological characters.

According to the 18 descriptors, the 11 varieties were clustered into four different groups, as shown in Figure 2. The first quadrant was made up of Magabari, Kigabali and Newkawogo. The second quadrant placed Rwabuganda, Kyabafuruki and Naspot I varieties together. In the third quadrant, Kyembadula, Dimbukabukulula, BND 145L and
Shock were grouped together. The fourth quadrant consisted of only one variety, Naspot II, as shown in Figure 2.

\section{Discussion}

The extent and distribution of the variation in sweet potato is essential for sound conservation strategies, whereby conservation and sustainable use of phenotypic resources is essential to meet the demand for future food security [5]. Assessment of morphological diversity and relationships among sweet potato varieties is very important, not only for germplasm conservation, but also for breeding purposes, especially during the selection of varieties having superior qualities [14]. This particular study, showed that there is a high level of phenotypic diversity exhibited in sweet potato, using both morphological characters and proximate composition. Among the varieties studied, most of the morphological characters were highly variable. This high variability in sweet potato genotypes has been previously reported by [15] and it is caused by natural mutations for such traits as root and skin colour, as well as leaf and vine characteristics.

In the early stages of germination, the general outline of the leaf allowed for separation of the seedlings as duals and non-duals. This character is very important in the selection of dual-purpose sweet potato because it is not affected by the environment [4]. Leaf outline in sweet potato has also been reported to be a major expression of the crop's diversity [5]. Selection of dual-purpose sweet potato is based on the root to biomass ratio (i.e., root / vine ratio) targeting those varieties that maximise the production of vines while maintaining good productivity of tuberising roots [7].

Dual-purpose sweet potato allows a low number of toppings, which enables spreading the availability of fodder over the year without significantly affecting root yields [12]. In this study, the varieties selected were all spreading types with high ground cover, which is an indication of fodder availability. There was high variation in all the leaf characters and vine characters evaluated among the varieties, except in vine-internode length (VIL) and vine tip pubescence (VTP). This is because these characters are influenced by environmental factors. The high variability of vegetative characters among the varieties can be attributed to the high polyploidy level in sweet potato. This variation was also shown in the dendrogram in which Naspot I and Rwabuganda clustered together as dual varieties. Naspot II stands out on its own. Principal correlation analyses 1 and 2 were the main contributors to variation among the varieties. Twining, Plant Type, Groundcover, Vine Internode Length, Node Diameter, Vine Pigmentation, Vine Tip Pubescence, Vine Weight, Abaxial Pigmentation, Immature Leaf Pigmentation, Petiole Length, Petiole Pigmentation, Root Cortex Thickness, Root Skin Colour and Root Weight were the main traits contributing to this variation.

\section{Conclusion}

Morphologically, based on the root / forage ratio, Naspot I is a high dual variety, Rwabuganda and New Kawogo are low dual varieties and BND145L, Shock, Dimbuka bukulula, Kigabali, Magabari, Naspot II, Kyabafuruki and Kyebandula are forage varieties. Based on this study, it is recommended that the variety Naspot I could be grown to help resolve food versus feed competition in smallholder crop-livestock operations between humans and livestock. This is because the vines and roots of Naspot I are suitable as feed for animals and the roots are marketable for direct consumption by humans. This study could also help recommend to farmer show dual-purpose sweet potato could 
Citation: Mbithe MJ, Steven R, Agili S, Kivuva MB, Kioko WF, et al. (2016) Morphological Characterisation of Selected Ugandan Sweet potato (Ipomoea batatas L) Varieties for Food and Feed. J Phylogen Evolution Biol 4: 163. doi:10.4172/2329-9002.1000163

be produced, in order to provide enough healthier food to millions of children in Uganda and in the world, and better feed for live-stock farmers.

\section{References}

1. Salami A, Kamara AB, Brixiova Z, Bank AD (2010) Small holder Agriculture in East Africa: Trends, Constraints and Opportunities. Working Paper No. 105

2. Chauvin ND, Mulangu F, Porto G (2012) Food Production and Consumption Trends in Sub-Saharan Africa: Prospects for the Transformation of the Agricultural Sector 1-76.

3. Sinha AK, Mishra PK, Uri V (2013) Morphology based multivariate analysis of phenotypic diversity of landraces of rice (Oryza sativa L.) of Bankura district of West Bengal. Journal of Crop and Weed 9: 115-121.

4. Huaman Z (1999) Sweet potato germplasm management (Ipomea batatas) Training manual 218

5. Karuri HW, Ateka EM, Amata R, Nyende AB, Muigai AWT, et al. (2010) Evaluating diversity among Kenyan sweet potato genotypes using morphological and SSR markers. Int J Agric Biol 12: 33-38.

6. Huaman Z (1991) Descriptors for Sweet potato. International Board for Plant Genetic Resources, Rome, Italy.

7. León-Velarde CU (2000) Using Competing Traits to Select Dual-Purpose Sweet potato in Native Germplasm. CIP Program Report 289-294.
8. Burri BJ (2011) Evaluating Sweet Potato as an Intervention Food to Prevent Vitamin A Deficiency. Comprehensive Reviews in Food Science and Food Safety 10: 118-130.

9. Woolfe JA (1992) Sweet Potato: An Untapped Food Resource.

10. Antia BS, Akpan EJ, Okon PA, Umoren IU (2006) Nutritive and Anti-Nutritive Evaluation of Sweet Potatoes (Ipomoea batatas) Leaves. Pakistan Journal of Nutrition 5: 166-168.

11. Nyaata OZ, Dorward PT, Keatinge JDH, O'Neill MK (2000) Availability and use of dry season feed resources on small holder dairy farms in central Kenya. Agroforestry Systems 50: 315-331.

12. Claessens L, Stoorvogel JJ, Antle JM (2008) Ex ante assessment of dualpurpose sweet potato in the crop-livestock system of western Kenya: A minimum-data approach. Agricultural Systems 99: 13-22.

13. Andrade M, Barker I, Cole D, Fuentes S, Grüneberg W, et al. (2009) Unleashing the potential of sweet potato in Sub-Saharan Africa: Current challenges and way forward 197

14. Laurie A, Thiart SM, Vorster HJ (2004) Sustainable production of root and tuber crops (potato, sweet potato, indigenous potato, cassava) in southern Africa. South African Journal of Botany 70: 60-66.

15. Maquia I, Muocha I, Naico A, Martins N, Gouveia M, et al (2013) Molecular morphological and agronomic characterization of the sweet potato (Ipomoea batatas L.) germplasm collection from Mozambique: Genotype selection for drought prone regions. South African Journal of Botany 88: 142-151. 